\title{
APPLYING THE ME-INDEX AND THE HYPERBOLIC FACTOR TO THE $Q$-EXPONENTIAL DISCOUNT FUNCTION DEFORMED BY THE AMOUNT
}

\author{
SALVADOR CRUZ RAMBAUD \\ Departamento de Economía y Empresa, UNIVERSIDAD DE ALMERÍA, ESPAÑA \\ e-mail: scruz@ual.es \\ ISABEL MARÍA PARRA OLLER \\ Departamento de Economía y Empresa, UNIVERSIDAD DE ALMERÍA, ESPAÑA \\ e-mail: ipo244@ual.es \\ MARÍA DEL CARMEN VALLS MARTÍNEZ \\ Departamento de Economía y Empresa, UNIVERSIDAD DE ALMERÍA, ESPAÑA \\ e-mail: mcvalls@ual.es
}

\begin{abstract}
Most empirical evidence on choices involving dated rewards has found that the discount rate used in intertemporal choices by individuals is not constant, as suggested by Samuelson (1937). In effect, the discount rate can depend on both time (showing decreasing impatience, stationarity or increasing impatience) and amount (showing magnitude effect, separability or magnitude effect reversal). A discount function, able to explain almost all the stages of impatience with respect to time and amount, is the so-called $q$-exponential discount function deformed by the amount which has been recently incorporated into the ambit of econophysics. The main objective of this manuscript is to apply the MEindex and a new version of the hyperbolic factor to this novel discount function, thus proving its validity. These indexes are our proposal to measure the magnitude and the delay effects, and both have been inspired in the hyperbolic factor introduced by Rohde (2010).
\end{abstract}

Keywords: intertemporal choice; magnitude effect; decreasing impatience; ME-index; hyperbolic factor.

JEL classification: G4, G40.

\section{Una aplicación del EM-index y del factor hiperbólico a la función de descuento q- exponencial deformada por la cuantía}

\section{RESUMEN}

La mayor parte de la evidencia empírica sobre la elección de recompensas a lo largo del tiempo ha demostrado que la tasa de descuento utilizada no es constante, tal y como fue sugerido por Samuelson (1937). En efecto, la tasa de descuento puede depender del tiempo (pudiendo aparecer el efecto impaciencia decreciente, la estacionariedad o la impaciencia creciente) o de la cuantía (mostrando el efecto magnitud, la separabilidad o la reversión del efecto magnitud). Una función de descuento, capaz de explicar casi todos estos estados de impaciencia con respecto al tiempo y la cuantía, es la denominada función $q$-exponencial deformada por la cuantía, recientemente incorporada al ámbito de la Econofísica. El objetivo principal de este artículo es la aplicación del EM-index y de una nueva versión del factor hiperbólico a esta novedosa función de descuento, y así comprobar su validez. Estos índices constituyen nuestra propuesta para medir los efectos magnitud y plazo, y ambos están inspirados en el factor hiperbólico introducido por Rohde (2010).

Palabras clave: elección intertemporal; efecto magnitud; impaciencia decreciente; EM-index, factor hiperbólico.

Clasificación JEL: G4, G40.

Artículo recibido el 7 de mayo de 2019 y aceptado el 24 de mayo de 2019 Artículo disponible en versión electrónica en la página www.revista-eea.net ISSN 1697-5731 (online) - ISSN 1133-3197 (print) 


\section{INTRODUCTION}

The framework of this paper is intertemporal choice, a field derived from the classic economics aimed to explain how people make their future decisions involving dated rewards. The importance of this discipline in research has been increasing during last decades, because such decisions imply not only individual but also important social interests.

In 1937, Samuelson proposed the Discounted Utility (hereinafter, DU) model in the ambit of intertemporal choice. Such model is based on assuming that people discount future incomes at a constant discount rate, that is to say, the discount rate does not change when time or amount increases. Despite these limitations, the model received a strong support from economists, given its similarity to the compound interest, which led to its generalization as a normative model.

However, in the last decades, many empirical studies have emerged, showing the finding of different anomalies, that is to say, individual behaviours which contradict the setting proposed by the DU model. Among these anomalies, we can highlight the decreasing impatience and the magnitude effect.

On the one hand, decreasing impatience arises when an individual decides to choose a smaller, sooner better than a larger, later reward. However, if the delay corresponding to both amounts is increased by a same time period, they would probably choose the larger, later instead of the smaller, sooner amount. In this case, we say that a preference reversal or dynamic inconsistency takes place. For example, a person could prefer the receipt of $€ 50$ today rather than $€ 60$ in a month, but also she could prefer $€ 60$ in a year and a month rather than $€ 50$ in a year. Although the waiting time between both rewards is the same in both cases, viz a month, the reward is less preferred when time is closer than distant. Some works revealing such anomalies are Green et al. (1981), Green, Fristoe and Myerson (1994), Kirby and Herrnstein (1995) and Bocquého, Jacquet and Reynaud (2013). Some of the most well-known models used to explain the decreasing impatience and the dynamic inconsistency are the hyperbolic discount (Ainslie and Herrnstein, 1981; Harvey, 1986; Loewenstein and Prelec, 1992; Green, Fry and Myerson, 1994) and the quasi-hyperbolic function (Laibson, 1997; Prelec, 2004; Benhabib, Bisin and Schotter, 2010). Additionally, Rohde (2010) proposed a measure of this effect, the so-called hyperbolic factor.

On the other hand, the magnitude effect is one of the most robust anomalies presented by the DU model which means higher discount rates when discounting smaller than larger amounts. That is to say, the discount rate decreases as the reward amount increases. Obviously, a necessary condition for the existence of the magnitude effect is that the discount function, involved in the process of decision making, depends on the amount, and not only on the time. Said in other words, this anomaly can be only explained by using non-separable discount models (Killeen, 2009; Benhabib, Bisin and Schotter, 2010; Noor, 2011). For example, a person could prefer $€ 50$ now rather than $€ 100$ in one year, but also $€ 10,000$ in one year rather than $€ 5,000$ now. Although the relative gain is the same in both cases (50\%), people tend to be more patient towards the receipt of the larger amount. This effect was revealed by numerous empirical studies (Thaler, 1981; Green, Myerson and McFadden, 1997; Chapman and Winquist, 1998; Kirby, Petry and Bickel, 1999; Schoenfelder and Hantula, 2003; Estle et al., 2006; Benhabib, Bisin and Schotter, 2010; Andersen et al., 2013; Meyer, 2015).

A discount function which is able to explain both decreasing impatience and magnitude effect is the so-called q-exponential discount function deformed by amount, recently proposed by Cruz Rambaud, Parra Oller and Valls Martínez (2018). This function arises when applying a general methodology to obtaining non-separable functions to the q-exponential discount function proposed by Cajueiro (2006), resulting in:

$$
F(x, t)=\frac{x}{\left[1+k(1-q) t x^{\alpha}\right]^{1 /(1-q)}} ; q \in \mathfrak{R}-\{1\} ; \alpha \in \mathfrak{R} ; k \in \mathfrak{R}^{+} .
$$

Depending on the values of $\mathrm{k}, \mathrm{q}$ and $\alpha$, this function can explain different stages of inconsistency (decreasing impatience (DI), stationarity and increasing impatience (II)) and separability (magnitude effect (ME), separability and reverse magnitude effect), as displayed in Table 1. 
Table 1

\begin{tabular}{|c|c|c|c|}
\hline & $q<1-\alpha$ & $q=1-\alpha$ & $q>1-\alpha$ \\
\hline$\alpha \in(-\infty, 0)$ & ME \& DI & ME \& DI & ME \& II \\
\hline$\alpha=0$ & Separable \& DI & Separable \& DI & Separable \& II \\
\hline$\alpha \in(0,+\infty)$ & Reverse ME \& DI & Reverse ME \& DI & Reverse ME \& II \\
\hline
\end{tabular}
Source: Cruz Rambaud, Parra Oller and Valls Martínez (2018).

The $q$-exponential discount function is an extension of the generalized exponential function studied in nonextensive thermodynamics (Tsallis, 1994) and has a great importance in econophysics. Some of its limitations have been solved by the $q$-exponential discount function deformed by the amount. The main objective of this paper is to apply the ME-index and a new version of the hyperbolic factor to this novel discount function, thus proving its effectiveness in explaining the impatience with respect to the amount and the time. These indexes are our proposal to measure the magnitude and the delay effects, and both have been inspired in the hyperbolic factor introduced by Rohde (2010).

After this introduction, in Section 2, we introduce the concept of ME-index to detect the presence of the magnitude effect, by showing its use with several examples. Likewise, in Section 3, we apply this index to the $q$-exponential discount function deformed by the amount. Additionally, in sections 4 and 5, we present a new version of the hyperbolic factor (Rohde, 2010) by applying it to the aforementioned discount function. Finally, Section 6 summarizes and concludes.

\section{THE ME-INDEX}

The objective of this section is to provide a parameter, called the ME-index, able to detect and measure the presence of the magnitude effect by starting from two indifference pairs. To do this, assume that the preference relation $\succeq$ satisfies the axioms of weak order (reflexive, complete and transitive), monotonicity and continuity. Starting from the following pairs of indifference:

$$
(x, s) \sim(y, t) \text { and }(d x, s) \sim(u y, t),
$$

where $0<x<y$ and $0<s<t$, the $M E$-index is defined as follows:

$$
\text { ME - index := } \frac{(u-d) y}{u x-d y} \text {. }
$$

\subsection{Case in which the magnitude effect holds.}

The magnitude effect holds if $u<d$, which implies $u-d<0$. Moreover, as $x<y$, one has $u x<d y$ , which implies $u x-d y<0$. On the other hand, observe that, in this case, $u x-d y<u y-d y=(u-d) y<0$ . Therefore,

$$
\text { ME - index }=\frac{(u-d) y}{u x-d y}<\frac{(u-d) y}{(u-d) y}=1 .
$$

As, moreover, the ME-index is, in this case, positive, one has:

$$
0<\text { ME-index }<1 \text {. }
$$

Let us see an example.

Example 2.1. Let us calculate the ME-index with the following rewards: 
APPLYING THE ME-INDEX AND THE HYPERBOLIC FACTOR TO THE Q-EXPONENTIAL DISCOUNT FUNCTION DEFORMED BY THE AMOUNT

$$
(x, s) \Rightarrow(100,2) \text { and }(d x, s) \Rightarrow(200,2)
$$

where $d=2$. Firstly, we are going to calculate the rewards which are indifferent to the previous ones at instant $t=5$. To do this, we will use the non-separable discount function, proposed by Noor (2011) to explain the magnitude effect:

$$
x \delta^{s / x^{\alpha}}=y \delta^{t / y^{\alpha}}
$$

Based on the former equality, for $\delta=0.2$ and $\alpha=0.5$, we can obtain the amounts $y$ and $u y$ satisfying the following equivalences:

$$
(x, s) \sim(y, t) \Rightarrow(100,2) \sim(142.29,5)
$$

and

$$
(d x, s) \sim(u y, t) \Rightarrow(200,2) \sim(261.89,5)
$$

from where $u=1.840559717$, and so:

$$
\text { ME-index }=0.225670
$$

As the ME-index is located between 0 and 1, the magnitude effect holds

\subsection{Case in which the magnitude effect reversal holds.}

The magnitude effect reversal holds if $u>d$, which implies $u-d>0$. However, there is not a general relation between $u x$ and $d y$, so that the reversal of the magnitude effect can appear in two different ways, depending on such relation:

2.2.1. Case in which $u x<d y$.

If $u x<d y$ (or, equivalently, $1<\frac{u}{d}<\frac{y}{x}$ ), one has $u x-d y<0$ and, therefore,

$$
\text { ME-index }<0 \text {. }
$$

This magnitude effect reversal is said to be weak. Let us see an example.

Example 2.2. Let us calculate the ME-index with the following rewards:

$$
(x, s) \Rightarrow(10,1) \text { and }(d x, s) \Rightarrow(2,000 ; 1)
$$

where $d=200$. Firstly, we are going to calculate the rewards which are indifferent to the former ones at instant $t=3$. To do this, we will use the non-separable discount function proposed by Myerson and Green (1995) which does not satisfy the magnitude effect, giving rise to the following equation:

$$
(x-h) e^{-k s}+h=(y-h) e^{-k t}+h .
$$


Based on the former equality, for $k=0.5$ and $h=5$, we can obtain the amounts $y$ and $u y$ satisfying the following equivalences:

$$
(x, s) \sim(y, t) \Rightarrow(10,1) \sim(18.59,3)
$$

and

$$
(d x, s) \sim(u y, t) \Rightarrow(2,000 ; 1) \sim(5,427.97 ; 3),
$$

from where $u=291.9612875$, and so:

ME-index $=-2.141267$.

As the ME-index is less than 0, the weak magnitude effect reversal holds.

2.2.2. Case in which $u x>d y$.

If $u x>d y \quad$ (o, equivalently, $1<\frac{y}{x}<\frac{u}{d}$ ), the same as the paragraph 2.1, we can put $0<u x-d y<u y-d y=(u-d) y$. Therefore,

$$
\mathrm{ME}-\text { index }=\frac{(u-d) y}{u x-d y}>\frac{(u-d) y}{(u-d) y}=1 .
$$

Thus, in this case, one has:

$$
\text { ME - index }>1 \text {. }
$$

This magnitude effect reversal is said to be moderate. Let us see an example.

Example 2.3. Let us calculate the ME-index with the following rewards:

$$
(x, s) \Rightarrow(0.5,2) \text { and }(d x, s) \Rightarrow(0.75,2),
$$

where $d=1.5$. Firstly, we are going to calculate the rewards which are indifferent to the former ones at instant $t=2.1$. To do this, we will use the following non-separable discount function which does not satisfy the magnitude effect, giving rise to the following equation:

$$
\frac{x}{\sqrt{x^{2} s^{3}+1}}=\frac{y}{\sqrt{y^{2} t^{3}+1}}
$$

Based on the former equality, we can obtain the amounts ${ }^{y}$ and $u y$ satisfying the following equivalences:

$$
(x, s) \sim(y, t) \Rightarrow(0.5,2) \sim(0.6,2.1)
$$

and

$$
(d x, s) \sim(u y, t) \Rightarrow(0.75,2) \sim(1.39,2.1),
$$

from where $u=2.3$, and so:

$$
\mathrm{ME}-\text { index }=1.92 \text {. }
$$

As the ME-index is greater than 1, the moderate magnitude effect reversal holds. 
Table 2 summarizes the different cases analyzed in this section.

Table 2.

Different types of magnitude effect according to the ME-index value.

\begin{tabular}{|c|c|c|c|}
\hline Magnitude effect & \multicolumn{2}{|c|}{ Magnitude effect reversal } & Separability \\
\hline $0<$ ME-index $<1$ & ME-index $<0$ & ME-index $>1$ & ME-index $=0$ \\
\hline--- & Weak & Moderate & --- \\
\hline
\end{tabular}

\section{APPLYING THE ME-INDEX TO THE $Q$-EXPONENTIAL FUNCTION DEFORMED BY THE}

\section{AMOUNT}

After seeing in Section 2 how the ME-index works, it is time to apply this index to the intertemporal decisions governed by a $q$-exponential discount function deformed by the amount (Cruz Rambaud, Parra Oller and Valls Martínez, 2018). To do this, we are going to consider the different stages of separability that this function can represent depending on the value of parameter $\alpha$.

\subsection{Case in which $\alpha \in(-\infty, 0)$.}

For these values, the q-exponential discount function deformed by the amount explains the magnitude effect (see Table 1), then the ME-index must be located in the interval $(0,1)$. Let us see a confirming example.

Example 3.1. Let us calculate the ME-index with the following rewards:

$$
(x, s) \Rightarrow(60,1) \text { and }(d x, s) \Rightarrow(1,200 ; 1),
$$

where $d=20$. Firstly, we are going to calculate the rewards which are indifferent to the former ones at instant $t=3$. To do this, we will use the $q$-exponential discount function deformed by the amount, giving rise to the following equation:

$$
\frac{x}{\left[1+k(1-q) s x^{\alpha}\right]^{1 /(1-q)}}=\frac{y}{\left[1+k(1-q) t y^{\alpha}\right]^{1 /(1-q)}},
$$

Based on the former equality, for $\alpha=-0.15, k=0.3$ and $q=-2$, we can obtain the amounts $y$ and uy satisfying the following equivalences:

$$
(x, s) \sim(y, t) \Rightarrow(60,1) \sim(70.63041,3)
$$

and

$$
(d x, s) \sim(u y, t) \Rightarrow(1,200 ; 1) \sim(1,361.576 ; 3),
$$

from where $u=19.2775$, and so:

$$
\text { ME-index }=0.199376
$$

As the ME-index is located between 0 and 1, the magnitude effect holds. Then, the ME-index confirms the fact that this function explains the magnitude effect for negative values of $\alpha$. 
3.2. Case in which $\alpha=0$.

For this value, the $q$-exponential discount function deformed by the amount is separable, then the ME-index must be equal to 0 . Let us see a confirming example.

Example 3.2. Let us calculate the ME-index with the following rewards:

$$
(x, s) \Rightarrow(50,3) \text { and }(d x, s) \Rightarrow(500 ; 3),
$$

where $d=10$. Based on the equality used in Example 3.1, for $\alpha=0, k=0.3$ and $q=-0.225523$, we can obtain the amounts $y$ and ${ }^{u y}$, at instant $t=5$, satisfying the following equivalences:

$$
(x, s) \sim(y, t) \Rightarrow(50,3) \sim(63.86,5)
$$

and

$$
(d x, s) \sim(u y, t) \Rightarrow(500,3) \sim(638.60,5) .
$$

from where $u=10$, and so:

$$
\text { ME }- \text { index }=0 .
$$

Then, the ME-index confirms the separability of this function when $\alpha=0$.

\subsection{Case in which $\alpha \in(0, \infty)$.}

For these values, the q-exponential discount function deformed by the amount explains the reversal of magnitude effect, then the ME-index must be either negative or greater than 1 . Let us see a confirming example.

Example 3.3. Let us calculate the ME-index with the following rewards:

$$
(x, s) \Rightarrow(3,2) \text { and }(d x, s) \Rightarrow(600,2),
$$

where $d=200$. Based on the equality used in Example 3.1, for $\alpha=2.383384, k=0.3$ and $q=-4$, we obtain the amounts ${ }^{y}$ and ${ }^{u y}$, at instant $t=4$, satisfying the following equivalences:

$$
(x, s) \sim(y, t) \Rightarrow(3,2) \sim(3.8839,4)
$$

and

$$
(d x, s) \sim(u y, t) \Rightarrow(600,2) \sim(885.8431,4)
$$

from where $u=228.08$, and so:

$$
\text { ME-index }=-1.178582 \text {. }
$$

As the ME-index is negative, the reversal of magnitude effect holds. Then, the ME-index confirms the fact that this function explains the reversal of magnitude effect when $\alpha \in(0, \infty)$.

\section{THE HYPERBOLIC FACTOR}

In this section, we are going to present an improvement of the so-called hyperbolic factor introduced by Rohde (2010). This factor measures the decreasing impatience in intertemporal choice. 
Consider $x, y \in X$, where $X=\mathfrak{R}^{m}$, the set of possible incomes, and $s, t, \tau, \sigma \in T$, where $T=\mathfrak{R}^{+}$, the set of possible instants involved in the intertemporal choice. As in Section 2, let us suppose that the preference $\succeq$ satisfies the axioms of weak order (reflexive, transitive and complete), monotonicity and continuity. However, assume that the preference $\succeq$ does not satisfy the axiom of stationarity since it shows decreasing impatience. This means that, for all $s<t, \tau \in T$,

- $\quad 0<x<y$ and $(x, s) \sim(y, t)$ implies $(x, s+\tau) \preceq(y, t+\tau)$, and

- $\quad x<y<0$ and $(x, s) \sim(y, t)$ implies $(x, s+\tau) \succeq(y, t+\tau)$.

The absence of the stationarity axiom shows that time differences tend to be less important, when these occur in the distant future.

By considering again the equivalent rewards $(x, s) \sim(y, t)$, where $s<t$, and by assuming that $(x, s+\sigma) \sim(y, t+\tau)$, decreasing impatience implies that $\tau-\sigma>0$ which, obviously, is a measure of decreasing impatience. However, Rohde (2010) proposes another measure which allows to distinguish the different stages of impatience and which remains constant for all models related to hyperbolic discounting. This measure is the so-called hyperbolic factor which is defined starting from two pairs of indifferences $(x, s) \sim(y, t)$ and $(x, s+\sigma) \sim(y, t+\tau)$, in the following way:

$$
H(s, t, \sigma, \tau):=\frac{\tau-\sigma}{t \sigma-s \tau} .
$$

Nevertheless, in this paper we are going to introduce an improvement of the hyperbolic factor which will facilitate the subsequent analysis, as follows:

$$
H^{*}(s, t, \sigma, \tau):=\frac{t(\tau-\sigma)}{t \sigma-s \tau} .
$$

\subsection{Case in which the impatience is increasing.}

Increasing impatience holds if $\tau<\sigma$, which implies $\tau-\sigma<0$. Moreover, as $s<t$, one has $s \tau<t \sigma$, which implies $t \sigma-s \tau>0$. Therefore,

$$
H^{*}(s, t, \sigma, \tau)<0 \text {. }
$$

On the other hand, observe that, in this case, ${ }^{t \sigma-s \tau>t \sigma-t \tau=t(\sigma-\tau)>0}$. Therefore,

$$
H^{*}(s, t, \sigma, \tau)=\frac{t(\tau-\sigma)}{t \sigma-s \tau}>\frac{t(\tau-\sigma)}{t(\sigma-\tau)}=-1 .
$$

Thus, in this case, one has:

$$
-1<H^{*}(s, t, \sigma, \tau)<0 .
$$

Let us see an example.

Example 4.1. Let us calculate the hyperbolic factor with the following rewards:

$$
(x, s) \sim(y, t) \Rightarrow(x, 10) \sim(10,15) .
$$

Firstly, we are going to calculate the reward $x$ to complete the former indifference. To do this, we will use the linear discount function, giving rise to the following equation: 


$$
x(1-i s)=y(1-i t)
$$

Based on the previous equality, for $i=0.05$, we can obtain $x=5$, remaining:

$$
(x, s) \sim(y, t) \Rightarrow(5,10) \sim(10,15)
$$

Now, consider the following indifference, where $\tau=2$ :

$$
(x, s+\sigma) \sim(y, t+\tau) \Rightarrow(5,10+\sigma) \sim(10,15+2) .
$$

Using the former linear equation, the parameter $\sigma$ can be calculated, resulting in:

$$
(x, s+\sigma) \sim(y, t+\tau) \Rightarrow(5,10+4) \sim(10,15+2)
$$

Therefore,

$$
H^{*}(10,15,4,2)=-0.75<0
$$

As the hyperbolic factor is negative, the impatience is increasing.

\subsection{Case in which the impatience is decreasing.}

Decreasing impatience holds if $\tau>\sigma$, which implies $\tau-\sigma>0$. However, in this case, there is not a general relationship between $s \tau$ and $t \sigma$.

\subsubsection{Case in which $s \tau<t \sigma$.}

If $s \tau<t \sigma$ (or, equivalently, $1<\frac{\tau}{\sigma}<\frac{t}{s}$ ), then $0<\tau-\sigma<\frac{\tau(t-s)}{t}$. Therefore,

$$
H^{*}(s, t, \sigma, \tau)>0 \text {. }
$$

In this case, we will say that the impatience is moderately decreasing.

Let us see an example.

Example 4.2. Let us calculate the hyperbolic factor with the following rewards:

$$
(x, s) \sim(y, t) \Rightarrow(x, 1) \sim(20,3) .
$$

Firstly, we are going to calculate the reward $\mathrm{x}$ to complete the former indifference. To do this, we will use the generalized hyperbolic discount function introduced by Loewenstein and Prelec (1992), giving rise to the following equation:

$$
x(1+h s)^{-r / h}=y(1+h t)^{-r / h}, \text { where } h>0, r>0 .
$$

Based on the previous equality, for $h=0.5$ and $r=0.2002252$, we can obtain that $x=16.30$, remaining:

$$
(x, s) \sim(y, t) \Rightarrow(16.30,1) \sim(20,3)
$$

Now, consider the following indifference, where $\tau=1$ :

$$
(x, s+\sigma) \sim(y, t+\tau) \Rightarrow(16.30,1+\sigma) \sim(20,3+1) .
$$

Using the former equation, the parameter $\sigma$ can be calculated, resulting in:

$$
(x, s+\sigma) \sim(y, t+\tau) \Rightarrow(16.30,1+0.6) \sim(20,3+1)
$$

Therefore, 
APPLYING THE ME-INDEX AND THE HYPERBOLIC FACTOR TO THE Q-EXPONENTIAL DISCOUNT FUNCTION DEFORMED BY THE AMOUNT

$$
H^{*}(1,3,0.6,1)=1.5>0
$$

As the hyperbolic factor is positive, the impatience is moderately decreasing.

4.2.2. Case in which $s \tau>t \sigma$.

If $s \tau>t \sigma$ (or, equivalently, $\frac{\tau}{\sigma}>\frac{t}{s}>1$ ), then $\tau-\sigma \geq \tau \frac{(t-s)}{t}>0$. Therefore,

$$
H^{*}(s, t, \sigma, \tau)<0 .
$$

But observe that, in this case, $0>t \sigma-s \tau>t \sigma-t \tau=t(\sigma-\tau)$. Therefore,

$$
H^{*}(s, t, \sigma, \tau)=\frac{t(\tau-\sigma)}{t \sigma-s \tau}<\frac{t(\tau-\sigma)}{t(\sigma-\tau)}=-1 .
$$

Therefore, in this case, one has:

$$
H^{*}(s, t, \sigma, \tau)<-1 .
$$

In this case, we will say that the impatience is strongly decreasing.

\subsection{Case in which the impatience is constant.}

Finally, if the impatience is constant then the hyperbolic factor is equal to 0 :

$$
H^{*}(s, t, \sigma, \tau)=0 \text {. }
$$

Let us see an example.

Example 4.3. Let us calculate the hyperbolic factor with the following rewards:

$$
(x, s) \sim(y, t) \Rightarrow(x, 2) \sim(100,5) .
$$

Firstly, we are going to calculate the reward $\mathrm{x}$ to complete the former indifference. To do this, we will use the exponential discount function, giving rise to the following equation:

$$
y e^{-r t}=x e^{-r s}
$$

Based on the previous equality, for $r=0.5$, we can obtain that $x=22.31$, remaining:

$$
(x, s) \sim(y, t) \Rightarrow(22.31,2) \sim(100,5)
$$

Now, consider the following indifference, where $\tau=2$ :

$$
(x, s+\sigma) \sim(y, t+\tau) \Rightarrow(22.31,2+\sigma) \sim(100,5+2) .
$$

Using the former equation, the parameter $\sigma$ can be calculated, resulting in:

$$
(x, s+\sigma) \sim(y, t+\tau) \Rightarrow(22.31,2+2) \sim(100,5+2)
$$

Therefore,

$$
H^{*}(2,5,2,2)=0
$$

As the hyperbolic factor is zero, the impatience is constant. 
Table 3 summarizes the different cases analyzed in this section.

Table 3

Different levels of impatience with respect to the hyperbolic factor.

\begin{tabular}{|c|c|c|c|}
\hline $\begin{array}{c}\text { Increasing } \\
\text { Impatience (II) }\end{array}$ & \multicolumn{2}{|c|}{$\begin{array}{c}\text { Decreasing } \\
\text { Impatience (DI) }\end{array}$} & $\begin{array}{c}\text { Constant } \\
\text { Impatience }\end{array}$ \\
\hline$-1<H^{*}(s, t, \sigma, \tau)<0$ & $H^{*}(s, t, \sigma, \tau)>0$ & $H^{*}(s, t, \sigma, \tau)<-1$ & $H^{*}(s, t, \sigma, \tau)=0$ \\
\hline--- & Moderately DI & Strongly DI & --- \\
\hline
\end{tabular}
Source: Own elaboration.

\section{APPLYING THE NEW HYPERBOLIC FACTOR TO THE $Q$-EXPONENTIAL FUNCTION DEFORMED BY THE AMOUNT}

After defining a new version of the hyperbolic factor introduced by Rohde (2010), and analogously to Section 3, we are going to apply this novel index to the $q$-exponential discount function deformed by the amount (Cruz Rambaud, Parra Oller and Valls Martínez, 2018). In this case, we are going to consider the different stages of impatience that this function can achieve depending on the values of $q$.

\subsection{Case in which $q \leq 1-\alpha$.}

For these values, the q-exponential discount function deformed by the amount explains decreasing impatience, then the hyperbolic factor must be positive or less than -1. Let us see a confirming example.

Example 5.1. Let us calculate the hyperbolic factor with the following rewards:

$$
(x, s) \sim(y, t) \Rightarrow(x, 3) \sim(50,5) .
$$

Firstly, we are going to calculate the reward $\mathrm{x}$ to complete the former indifference. To do this, we will use the $q$-exponential discount function deformed by the amount, giving rise to the following equation:

$$
\frac{x}{\left[1+k(1-q) s x^{\alpha}\right]^{1 /(1-q)}}=\frac{y}{\left[1+k(1-q) t y^{\alpha}\right]^{1 /(1-q)}}
$$

Based on the previous equality, for $\alpha=-3, k=0.3$ and $q=-2$, we can obtain that $x=49.99976$, remaining:

$$
(x, s) \sim(y, t) \Rightarrow(49.99976,3) \sim(50,5)
$$

Now, consider the following indifference, where $\tau=2$ :

$$
(x, s+\sigma) \sim(y, t+\tau) \Rightarrow(49.99976,3+\sigma) \sim(50,5+2) .
$$

Using the former equation, the parameter $\sigma$ can be calculated, resulting in:

$$
(x, s+\sigma) \sim(y, t+\tau) \Rightarrow(49.99976,3+1.998853) \sim(50,5+2)
$$

Therefore,

$$
H^{*}(3,5,1.99,2)=0.001435>0
$$

Thus, the value of hyperbolic factor confirms that this function explains the (moderately) decreasing impatience when $q \leq 1-\alpha$. 


\subsection{Case in which $q>1-\alpha$.}

For these values, the q-exponential discount function deformed by the amount explains increasing impatience, then the hyperbolic factor must be in the interval $(-1,0)$. Let us see a confirming example.

Example 5.2. Let us calculate the hyperbolic factor with the following rewards:

$$
(x, s) \sim(y, t) \Rightarrow(x, 7) \sim(100,10) .
$$

Based on the same equality as Example 5.1, for $\alpha=-3, k=0.3$ and $q=-1$, we can obtain that $x=99.99991$, remaining:

$$
(x, s) \sim(y, t) \Rightarrow(99.99991,7) \sim(100,10)
$$

Now, consider the following indifference, where $\tau=5$ :

$$
(x, s+\sigma) \sim(y, t+\tau) \Rightarrow(99.99991,7+\sigma) \sim(100,10+5) .
$$

Using the equation of Example 5.1, the parameter $\sigma$ can be calculated, resulting in:

$$
(x, s+\sigma) \sim(y, t+\tau) \Rightarrow(99.99991,7+5.003626) \sim(100,10+5)
$$

Therefore,

$$
H^{*}(7,10,5.003626,5)=-0.00241<0
$$

Thus, the value of hyperbolic factor confirms that this function explains the increasing impatience when $q>1-\alpha$.

\section{CONCLUSION}

The objective of this manuscript has been to apply the ME-index, our proposal to measure the magnitude effect, to the $q$-exponential discount function deformed by the amount, recently emerged in the field of econophysics. This function is able to explain different stages of impatience respect to the amount (magnitude effect, separability and magnitude effect reversal), as well as different stages of impatience with respect to time (decreasing and increasing impatience).

To do this, in Section 2, we have introduced the ME-index as a measure arising from two pairs of indifference. This index is inspired in the hyperbolic factor introduced by Rohde (2010). Indeed, it provides a characterization of some non-separable models, as the generalized exponential function of Noor (2011) for which this index presents values between 0 and 1, being zero for those models which present separable amount and exponential time. Finally, if the index is negative or greater than 1, the intertemporal choices exhibit the magnitude effect reversal.

In Section 3, we have applied the ME-index to the $q$-exponential discount function deformed by the amount, proposed by Cruz Rambaud, Parra Oller and Valls Martínez (2018). In effect, we prove that this function can explain the different stages of impatience with respect to the amount starting from two indifference pairs, depending on the values of parameter ${ }^{\alpha}$.

Additionally, in Section 4, we have presented an improvement of the hyperbolic factor introduced by Rohde (2010) by explaining decreasing impatience when the new parameter is less than -1 or greater than 0 . Moreover, it explains increasing impatience when the hyperbolic factor is between -1 and 0 . Finally, when the discount rate is constant with respect to time, the hyperbolic factor is zero.

In Section 5, we have applied our new version of the hyperbolic factor to the $q$-exponential discount function deformed by the amount (Cruz Rambaud, Parra Oller and Valls Martínez, 2018). In this way, we have proved that, in effect, this function explains decreasing impatience when $q \leq 1-\alpha$, 
independently of the values of parameter $\alpha$. Likewise, it also explains increasing impatience when $q>1-\alpha$

Along this paper, we have shown that it is possible to prove the explicative validity of a discount function, specifically the q-exponential discount function deformed by the amount, by using these two indexes: the ME-index (impatience with amount) and the new hyperbolic factor (impatience with time).

\section{BIBLIOGRAPHIC REFERENCES}

AINSLIE, G., \& HERRNSTEIN, R. J. (1981). "Preference reversal and delayed reinforcement". Animal Learning and Behavior, 9, pp. 476-482. http://doi.org/10.3758/ BF03209777

ANDERSEN, S., HARRISON, G. W., LAU, M. I., \& RUTSTRÖM, E. E. (2013). "Discounting behaviour and the magnitude effect: Evidence from a field experiment in Denmark". Economica, 80, pp. 670-697. http://doi.org/10.1111/ecca.12028

BENHABIB, J., BISIN, A., \& SCHOTTER, A. (2010). "Present-bias, quasi-hyperbolic discounting, and fixed costs". Games and Economic Behavior, 69(2), pp. 205-223. http://doi.org/10.1016/j.geb.2009.11.003

BOCQUÉHO, G., JACQUET, F., \& REYNAUD, A. (2013). "Reversal and magnitude effects in long-term time preferences: Results from a field experiment". Economics Letters, 120, pp. 108-111. http://doi.org/10.1016/j.econlet.2013.04.006

CAJUEIRO, D. O. (2006). "A note on the relevance of the $q$-exponential function in the context of intertemporal choices". Physica A: Statistical Mechanics and Its Applications, 364, pp. 385-388.

CHAPMAN, G. B., \& WINQUIST, J. R. (1998). "The magnitude effect: Temporal discount rates and restaurant tips". Psychonomic Bulletin \& Review, 5(1), pp. 119-123. http://doi.org/10.3758/BF03209466

CRUZ RAMBAUD, S., PARRA OLLER, I. M., \& VALLS MARTÍNEZ, M. C. (2018). "The amount-based deformation of the $q$-exponential discount function: A joint analysis of delay and magnitude effects". Physica A: Statistical Mechanics and Its Applications, 508, pp. 788-796. http://doi.org/10.1016/j.physa.2018.05.152

ESTLE, S. J., GREEN, L., MYERSON, J., \& HOLT, D. D. (2006). "Differential effects of amount on temporal and probability discounting of gains and losses". Memory \& Cognition, 34(4), pp. 914-928. http://doi.org/10.3758/BF03193437

GREEN, L., FRISTOE, N., \& MYERSON, J. (1994). "Temporal discounting and preference reversals in choice between delayed outcomes". Psychonomic Bulletin \& Review, 1(3), pp. 383-389.

GREEN, L., FRY, A. F., \& MYERSON, J. (1994). "Discounting of delayed rewards: A life-span comparison". Psychological Science, 5(1), pp. 33-36. http://doi.org/10.1111/j.1467-9280.1994.tb00610.x

GREEN, L., MYERSON, J., \& MCFADDEN, E. (1997). "Rate of temporal discounting decreases with amount of reward". Memory \& Cognition, 25(5), pp. 715-723. http://doi.org/10.3758/BF03211314

HARVEY, C. M. (1986). "Value functions for infinite-period planning". Management Science, 32(9), pp. 1123-1139. http://doi.org/10.2307/2632084

KILLEEN, P. R. (2009). "An additive-utility model of delay discounting". Psychological Review, 116(3), pp. 602619. http://doi.org/10.1037/a0016414

KIRBY, K. N., \& HERRNSTEIN, R. J. (1995). "Preference reversals due to myopic discounting of delayed reward". Psychological Science, 6(2), pp. 83-89.

KIRBY, K. N., PETRY, N. M., \& BICKEL, W. K. (1999). "Heroin addicts have higher discount rates for delayed rewards than non-drug-using controls". Journal of Experimental Psychology: General, 128(1), pp. 78-87. http://doi.org/10.1037/0096-3445.128.1.78

LAIBSON, D. (1997). "Golden eggs and hyperbolic discounting". Quarterly Journal of Economics, 112(2), pp. 443477. http://doi.org/10.1162/003355397555253

LOEWENSTEIN, G., \& PRELEC, D. (1992). "Anomalies in intertemporal choice: evidence and an intepretation". Quarterly Journal of Economics, 107(2), pp. 573-597.

MEYER, A. G. (2015). "The impacts of elicitation mechanism and reward size on estimated rates of time preference". Journal of Behavioral and Experimental Economics, 58, pp. 132-148. http://doi.org/10.1016/j.socec.2015.08.002

MYERSON, J., \& GREEN, L. (1995). "Discounting of delayed rewards: Models of individual choice". Journal of the Experimental Analysis of Behavior, 64(3), pp. 263-76. http://doi.org/10.1901/jeab.1995.64-263

NOOR, J. (2011). "Intertemporal choice and the magnitude effect". Games and Economic Behavior, 72, pp. 255270. http://doi.org/10.1016/j.geb.2010.06.006

PRELEC, D. (2004). "Decreasing impatience: A criterion for non-stationary time preference and "hyperbolic" discounting". The Scandinavian Journal of Economics, 106(3), pp. 511-532. http://doi.org/10.1111/j.14679442.2004.00380.x

ROHDE, K. I. M. (2010). "The hyperbolic factor : A measure of time inconsistency". Journal of Risk and Uncertainty, 41, pp. 125-140. http://doi.org/10.1007/s11166-010-9100-2

SAMUELSON, P. A. (1937). "A note on measurement of utility". The Review of Economic Studies, 4(2), pp. 155161.

SCHOENFELDER, T. E., \& HANTULA, D. A. (2003). "A job with a future? Delay discounting, magnitude effects, and domain independence of utility for career decisions". Journal of Vocational Behavior, 62, pp. 43-55. http://doi.org/10.1016/S0001-8791(02)00032-5 
APPLYING THE ME-INDEX AND THE HYPERBOLIC FACTOR TO THE Q-EXPONENTIAL DISCOUNT FUNCTION DEFORMED BY THE AMOUNT

THALER, R. H. (1981). "Some empirical evidence on dynamic inconsistency". Economic Letters, 8, pp. $201-207$. http://doi.org/10.1016/0165-1765(81)90067-7

TSALLIS, C. (1994). "What are the numbers that experiments provide?". Química Nova, 17(6), pp. $468-471$. 